\title{
Extreme jungholozäne hygrische Klimaschwankungen in Mitteleuropa und ihre Folgen
}

\author{
Hans-Rudolf Bork \& Helga BorK *)
}

\begin{abstract}
Heavy rainfall, gully erosion, sheet erosion, soil profiles, Younger Holocene, late medieval (14. and 18. century)

North German Hills (Göttingen region), Lower Saxony

TK 25: Nr. 4426, 4427
\end{abstract}

\begin{abstract}
Kurzfassung: Zur Rekonstruktion extremer hygrischer mittelalterlicher und neuzeitlicher Witterungsereignisse wurden zahlreiche Bodenprofile analysiert. Als wichtigstes Ergebnis ist festzuhalten, daß in der ersten Hälfte des 14. Jahrhunderts an den Hängen und in den Talauen bis über $10 \mathrm{~m}$ tiefe Schluchten einrissen. Die Steilheit der Kerbenwände und die aus Rutschmassen bestehenden Kerbenfüllungen erlauben wesentliche Rückschlüsse auf die Eintiefungsgeschwindigkeit und damit auf die Niederschläge, die diesen Abtrag auslösten. Demnach verursachten wenige katastrophale Starkregen das von stärkster flächenhafter Bodenerosion begleitete Schluchtenreißen. Schriftquellen bestätigen diese Resultate. Der überwiegende Teil der spätmittelalterlichen Erosionsformen wurde in den folgenden Jahrhunderten durch schwach erosive Niederschläge verfüllt. Eine zweite, schwächere, auf extreme Starkregen zurückzuführende Zerschneidungsphase wurde für das 18. Jh. nachgewiesen.
\end{abstract}

[Extreme Rainfall Fluctuations During Younger Holocene in Central Europe and the Resulting Soil Erosion]

Abstract: To enable conclusions about rainfall events during the Younger Holocene and their consequences, numerable soil profiles were analyzed. The most important result is the gully formation during the first half of the 14th century. Gullies more than $10 \mathrm{~m}$ deep were cut into arable land on slopes and in valleys. The steepness of the gullies and their slide-mass fillings give essential informations concerning the rate of dissection and the rainfall events which caused the erosion. The extreme gully-cutting was accompanied by heavy sheet erosion. Both processes must have been caused by very few catastrophic rainfall events. These results are confirmed by ancient documents. Most gullies of the 14 th century were filled up during the following centuries by

*) Anschrift der Autoren: Dr. H.-R. BORK und H. BORK, Abteilung für Physische Geographie und Landschaftsökologie, Technische Universität, Langer Kamp 19c, D -3300 Braunschweig. slightly erosive rainfall events. A second though less severe phase of gully erosion caused by heavy rainfall occured in the 18 th century.

\section{Einführung}

Die $\mathrm{h} y \mathrm{~g} \mathrm{r}$ is c h e $\mathrm{n}$ Extremereignisse in historischer Zeit stehen im Vordergrund des vorliegenden Beitrages. Im Rahmen des Forschungsvorhabens „Bodenerosion in südniedersächsischen Lößgebieten" der Abt. f. Physische Geographie und Landschaftsökologie der TU Braunschweig haben wir seit 1978 das Ausmaß der historischen Bodenerosion (das Ergebnis vor allem extremer hygrischer Niederschlagsereignisse) analysiert. Im Untereichsfeld und im südwestlichen Harzvorland wurden über 800 Standorte untersucht. Zunächst erfolgte die Bilanzierung der jungholozänen Bodenerosion (vgl. BORK 1983). Demnach wurden während Mittelalter und Neuzeit im Mittel die obersten $232 \mathrm{~cm}$ des auf den beackerten Hängen Südniedersachsens anstehenden Bodens erodiert.

Bereits in der Anfangsphase der Untersuchungen wurde deutlich, daß sich im Jungholozän Phasen mit starker flächenhafter und linearer Bodenerosion mit erosionsarmen Zeiten abwechselten. Dieser Befund hat uns dazu veranlaßt, den zeitlichen Verlauf der historischen Bodenerosion genauer zu rekonstruieren. Feinstratigraphische Detailanalysen wurden an Bodenprofilen in Niedersachsen und Hessen ausgeführt.

\section{Komplexe Sediment-Boden-Aufschlüsse als Zeugnisse extremer hygrischer Witterungsereignisse}

Die für eine Rekonstruktion des jungholozänen Klimas relevanten Ergebnisse feinstratigraphischer 
Detailanalysen werden zunächst am Beispiel des Aufschlusses Rüdershausen und der archäologisch-paläoökologischen Grabung Drudevenshusen vorgestellt. Es schließt sich eine zusammenfassende Darstellung der aus diesen und zahlreichen weiteren Profilen für den mitteleuropäischen Raum abgeleiteten hygrischen jungholozänen Klimageschichte an.

\subsection{Profil Rüdershausen}

In den Jahren 1979 bis 1982 wurde der heute weitgehend verschüttete Aufschluß in der ehemaligen Lehmgrube Rüdershausen untersucht. Dieses etwa 22 km ostnordöstlich von Göttingen gelegene Profil (vgl. Abb. 1) ist detailliert in BORK (1983: 40-44, 59, Abb. 14, 15) beschrieben. Im folgenden werden daher nur die Ergebnisse mitgeteilt, die Schlüsse auf die jungholozäne hygrische Klimageschichte erlauben.

An der Basis des Profils stehen 4 bis $10 \mathrm{~m}$ unter der Geländeoberfläche fluviale Sedimente an. Im Hangenden folgt über $2 \mathrm{~m}$ mächtiger intensiv verbraunter Löß, rotsandstreifiger kalkhaltiger Löß und vereinzelte Relikte der in sandstreifigem Löß entwickelten holozänen Parabraunerde. In dieser Abfolge war eine über $10 \mathrm{~m}$ mächtige Kerbtalfüllung aufgeschlossen. Rutschmassen, gradiert geschichtete Sedimente und homogene, schwach humose Kolluvien plombieren den Erosionsriß. Zahlreiche Keramikfunde ermöglich-

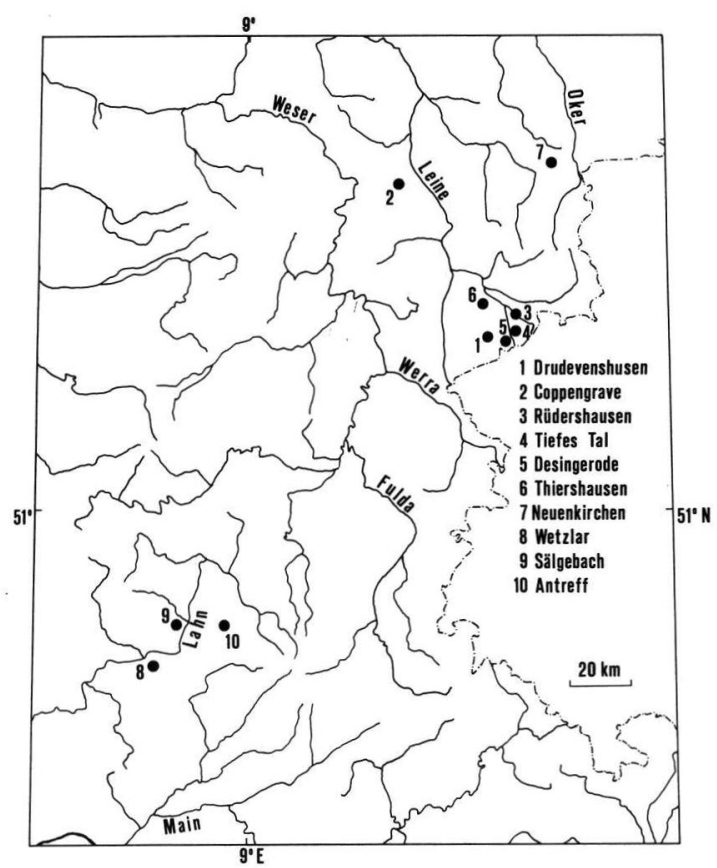

Abb. 1: Lage der datierten Profile. ten eine Datierung der unteren 5 bis $7 \mathrm{~m}$ der Kerbenfüllung in den Zeitraum vom 13. Jh. bis zur ersten Hälfte des 15. Jahrhunderts (Datierung durch H.-G. STEPHAN, Göttingen). Der obere Teil der Füllung sedimentierte in der frühen Neuzeit.

Die Umgebung des Aufschlusses wurde sehr wahrscheinlich im frühen Mittelalter gerodet und anschließend ackerbaulich genutzt. Dadurch wurde die holozäne geomorphodynamische Stabilitätsphase mit intensiver Bodenbildung beendet. Bis zum Beginn des Schluchtenreißens trat nur sehr schwacher flächenhafter Bodenabtrag auf. Beleg hierfür ist die Kerbenfüllung. So waren an der Basis der Kerbenfüllung zahlreiche Blöcke aus Al-Horizontmaterial des Holozänbodens aufgeschlossen, die nach der Einschneidung im obersten Bereich der Kerbenwände abgebrochen waren. Diese Blöcke wiesen Durchmesser bis zu $30 \mathrm{~cm}$ auf. Die beschriebenen Fakten beweisen, daß unmittelbar vor der Zerschluchtung der noch mindestens $30 \mathrm{~cm}$ mächtige Tonverarmungshorizont der holozänen Parabraunerde an der Geländeoberfläche lag. Geht man von einer mittleren Mächtigkeit von etwa $50 \mathrm{~cm}$ und einer mindestens 400jährigen landwirtschaftlichen Nutzung vor dem Schluchtenreißen aus, so ergibt sich ein durchschnittlicher jährlicher flächenhafter Bodenabtrag von maximal $8 \mathrm{t} / \mathrm{ha}$ im Bereich des Aufschlusses Rüdershausen. Dieser für einen relativ stark geneigten, konvexen Unterhang geringe Betrag weist auf eine erosionsarme Zeit hin. Es liegt daher nahe, auf lediglich schwach erosive Niederschlagsereignisse im frühen und hohen Mittelalter zu schließen.

Während des Spätmittelalters schnitt sich im Bereich des Aufschlusses Rüdershausen eine Kerbe mehr als $10 \mathrm{~m}$ tief in die pleistozänen Sedimente ein. In diesem Zusammenhang ist die Frage nach der Geschwindigkeit der Zerschneidung und damit nach der Anzahl und der Intensität der Niederschlagsereignisse zu stellen, die diesen Abtrag verursachten. Wesentliche Hinweise können Form und Füllung der Kerbe geben. Vor der Zerschneidung nahm eine flache Delle die Oberfläche ein. Starke lineare Erosion führte zur Ausbildung einer etwa $10 \mathrm{~m}$ tiefen und ebenso breiten Kerbe mit te il we is e ü be r h ä n g e n d e n W ä n d e n. Eine Detailaufnahme der Kerbenfüllung (vgl. BORK 1983: Abb. 14) - insbesondere der Rutschmassen - ermöglichte die genaue Rekonstruktion der Oberfläche. Die Feingliederung der pleistozänen Folge und die Differenzierung des holozänen Bodens in zahlreiche Subhorizonte erlaubten eine exakte Zuordnung insbesondere größerer Blöcke zu den Abbruchstellen an den Kerbenwänden. Die so rekonstruierte bzw. z. T. noch erhaltene ursprüngliche Steilheit der Kerbenwände erlaubt wesentliche Rückschlüsse auf die Zerschneidungsgeschwindigkeit. 
Beobachtungen an der unbewachsenen Aufschlußwand des Profils Rüdershausen in den Jahren 1979 bis 1985 zeigten, daß die aufgegrabene steile Profilwand im Verlauf der jeweils nächsten stärkeren Durchfeuchtung wieder zerstört wurde: Große Blöcke rutschten ab und noch im ersten Jahr nach einer Aufgrabung war das Profil wieder verstürzt. Während an trockenen Lößstandorten Steilwände oftmals über mehrere Jahre weitgehend unverändert erhalten bleiben, sind für Feuchtstandorte (Dellen und Kerben mit lateralem Bodenwasserzufluß) die für das Profil Rüdershausen beschriebenen Verhältnisse kennzeichnend. Aufschlußwand und spätmittelalterliche Kerbtalwand des Profils Rüdershausen ähnelten sich stark hinsichtlich Steilheit und Form. Wir können daher davon ausgehen, daß auch die steilen Kerbenwände im Spätmittelalter nur kurze Zeit standhielten. Wahrscheinlich vollzogen sich die Abbrüche und Rutschungen bereits während der ersten intensiveren Durchfeuchtung nach Bildung der Formen - somit vermutlich wenige Wochen bis Monate nach der Einschneidung. Diese rasche Plombierung beweist andererseits, daß die Einschneidung auf ein oder mehrere, kurz aufeinander folgende, kaum vorstellbar extreme Abflußereignisse und damit auf einen oder wenige Katastrophenregen zurückzuführen ist. Die extremen Starkregen der letzten Jahre hatten auch auf flurbereinigten und mit Rüben bewachsenen, großen Schlägen nur schwache flächenhafte und - mit Ausnahme vereinzelter bis zu $30 \mathrm{~cm}$ tiefer Rillen - keine lineare Bodenerosion zur Folge. Auf Hangstandorten, die im Mittelalter zerschluchtet wurden und die heute landwirtschaftlich genutzt werden, wurde im Beobachtungszeitraum (seit 1978) k e i n e lineare Bodenerosion registriert. Nur wenige Tiefenlinien, wie z. B. das Tiefe Tal bei Obernfeld, verzeichneten schwachen linienhaften $A b$ trag. Die rekonstruierte Geschwindigkeit und Intensität der Zerschluchtung sowie die heute an diesen Standorten auch bei Starkniederschlägen ausbleibende lineare Erosion weisen auf katastrophale spätmittelalterliche Niederschläge mit extrem hohen Intensitäten hin.

In der spätmittelalterlichen Kerbenfüllung waren neben Rutschmassen u. a. gradiert geschichtete Sedimente aufgeschlossen. Detailaufnahmen dieser Sedimente ergaben, daß nach der Einschneidung noch im Spätmittelalter mehrere Dutzend schwach erosive Niederschläge zur weitgehenden Plombierung des Erosionsrisses führten. Ein schwach humoses, nach Keramikdatierungen frühneuzeitliches Kolluvium schließt die Füllung des Kerbtales ab. In der unmittelbaren Umgebung wurden weitere verfüllte spätmittelalterliche Kerben gefunden.

Neben der spätmittelalterlichen konnte eine zweite, aber vergleichsweise weitaus schwächere, neuzeitliche
Zerschneidungsphase im Bereich des Profils Rüdershausen nachgewiesen werden. Es tieften sich bis zu 4,5 m tiefe Runsen ein. An der Basis einer Kerbe der zweiten Generation wurden in einem gradiert geschichteten Sediment zahlreiche Scherben entdeckt und in das 18. Jh. datiert. Im Hangenden waren Rutschmassen und humose, homogene Kolluvien aufgeschlossen. Obgleich die Wände dieser Runsen schwächer geneigt waren als die spätmittelalterlichen, deuten die Rutschmassen auf eine rasche Verfüllung und daher auf eine rasche Einschneidung sowie starke Niederschläge hin.

\subsection{Profilkomplex Drudevenshusen}

Eine genauere zeitliche Einordnung der ersten Zerschneidungsphase gelang für mehrere Profile, die im Bereich der Wüstung Drudevenshusen aufgegraben wurden. Die mittelalterliche Ortswüstung Drudevenshusen liegt etwa $13 \mathrm{~km}$ östlich von Göttingen, etwa $2 \mathrm{~km}$ südöstlich von Landolfshausen (zur Lage vgl. STEPHAN 1985; Abb. 5). Auf dem Gelände dieser Wüstung wurden in Zusammenarbeit mit dem Seminar für Ur- und Frühgeschichte der Universität Göttingen mehrere Grabungskampagnen durchgeführt (vgl. BORK 1985a: STEPHAN 1985). Die im Rahmen der hier diskutierten Fragestellung interessantesten Grabungsergebnisse sind in zwei Phasenzeichnungen dargestellt. Abb. 2 zeigt den Zustand eines über $80 \mathrm{~m}$ langen E-W-Profils zu vier verschiedenen Zeitpunkten. Die in neun Phasen gegliederte mittelalterliche Entwicklung des tiefenliniennahen Teils dieses Profils veranschaulicht Abb. 3.

Am Hang entwickelte sich im Holozän unter Wald in Löß eine Parabraunerde, die vor den mittelalterlichen Rodungen etwa 3 m mächtig war. In der Nähe der östlich der Wüstung gelegenen Tiefenlinie bildete sich eine (heute vergleyte) Schwarzerde-Parabraunerde.

Archäologische Befunde belegen, daß der Wüstungsbereich und das sich östlich anschließende Nebental der Suhle spätestens $800 \mathrm{n}$. Chr. gerodet und bebaut bzw. ackerbaulich genutzt wurde (vgl. STEPHAN 1985: 48). Dadurch wurde die holozäne geomorphodynamische Stabilitätszeit mit Bodenbildung beendet. Im Anschluß an die Rodungen wurden bis zum späten Hochmittelalter östlich der Siedlungsflächen am Unterhang (Abschnitt von 50 bis $74 \mathrm{~m}$ Profillänge, vgl. Abb. 2, 3) im Mittel die obersten $35 \mathrm{~cm}$ des Holozänbodens erodiert. Diesem Wert entspricht ein mittlerer jährlicher Abtrag von maximal $14 \mathrm{t} / \mathrm{ha}$ - ein niedriger Wert, der die Daten des Profils Rüdershausen bestätigt und damit ebenfalls auf ein erosionsund starkregenarmes Früh- und Hochmittelalter hinweist. Im 13. Jh. sedimentierte am Unterhang ein 

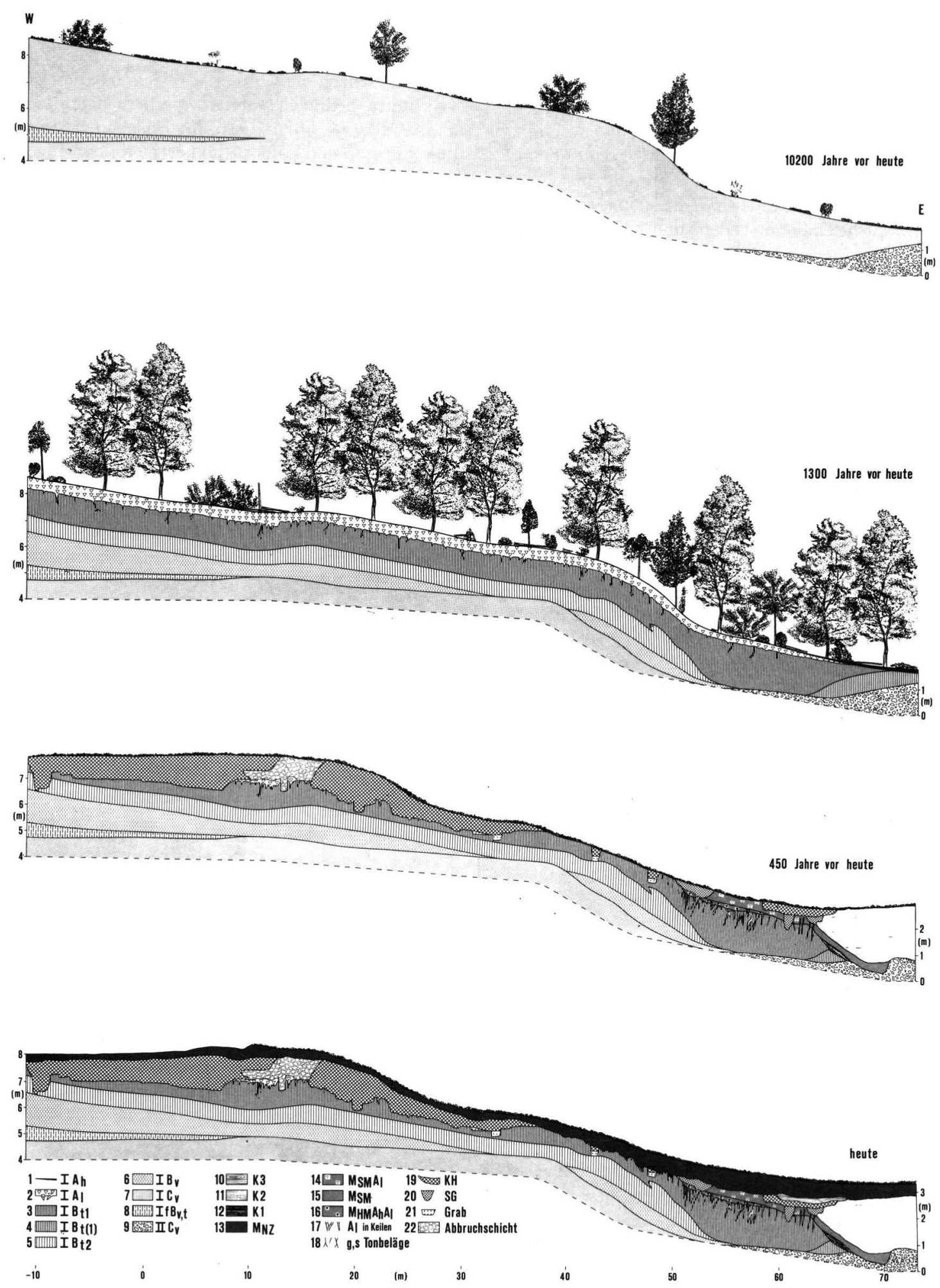

Abb. 2: E-W-Profil Drudevenshusen - jungholozäne Genese (2,5-fach überhöht). 

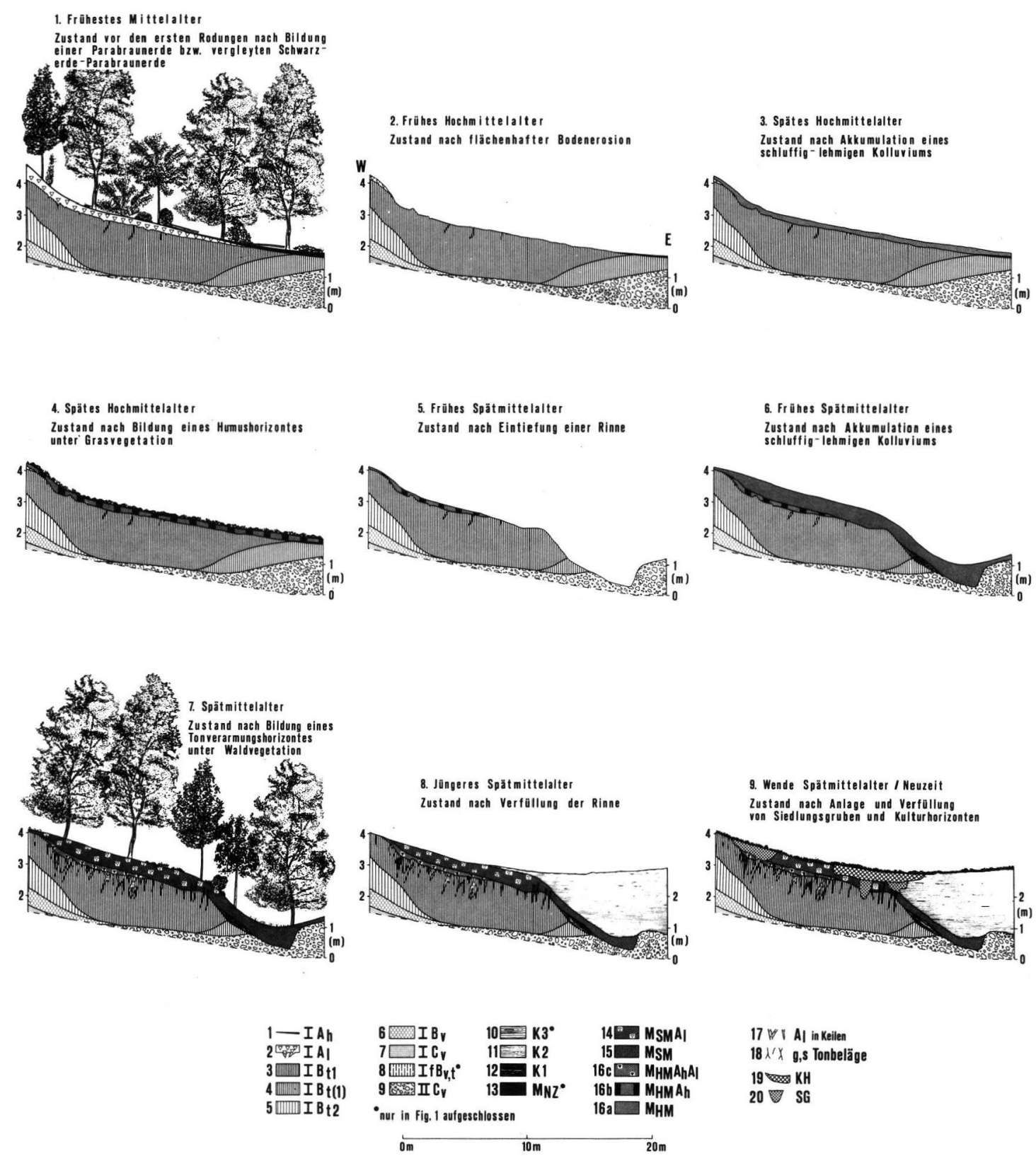

Abb. 3: E-W-Profil Drudevenshusen - mittelalterliche Genese im Bereich der Tiefenlinie (48,5-73 m Profillänge).

Kolluvium, in dem sich danach unter Grasvegetation ein Humushorizont ausbildete. Anschließend tiefte sich eine $12 \mathrm{~m}$ breite, im Bereich des E-W-Profils $2 \mathrm{~m}$ tiefe und bis in den heutigen Seulinger Wald hinaufreichende Kerbe ein (Zustand 5 in Abb. 3). Etwa $50 \mathrm{~m}$ talaufwärts besaß diese Kerbe eine Tiefe von über $3 \mathrm{~m}$, im Seulinger Wald lag dieser Wert bei mehr als $4 \mathrm{~m}$. Unmittelbar nach dem Kerbenreißen führte (nach Datierung umfangreichen Keramikmaterials durch H.-G. STEPHAN) im frühen 14. Jh. schwache flächenhafte Bodenerosion zur Akkumulation eines schluffig-lehmigen Kolluviums in der Rinne und ihrer Umgebung. Anschließend entwickelte sich in diesem Kolluvium ein Tonverarmungshorizont. Noch im Spätmittelalter wurde die Rinne mit geschichtetem Material vollständig plombiert (Zustand 8 in Abb. 3). Die Schichtung weist auf eine größere Zahl schwach erosiver Niederschlagsereignisse hin. Hinweise zur 
Tab. 1: Wüstung Drudevenshusen: Sedimente, Bodenhorizonte und Kulturschichten — Erläuterungen zu den Abb. 2 und 3

Nr. Horizont Charakterisierung

Schichtbez.

\begin{tabular}{|c|c|c|c|}
\hline 1 & I Ah & Humushorizont & \\
\hline 2 & I Al & Tonverarmungshorizont & Horizonte der vom \\
\hline 3 & I Bt 1 & kompakter Tonanreicherungshorizont & $\begin{array}{l}\text { Altholozän bis zum } \\
\text { Frühmittelalter }\end{array}$ \\
\hline 4 & I Bt (1) & verbraunter Horizont mit schwacher Tonanreicherung & unter Wald in Löß \\
\hline 5 & I Bt 2 & $\begin{array}{l}\text { verbraunter Horizont mit Tonanteicherung in } \\
\text { oberflächenparallelen Bändern }\end{array}$ & $\begin{array}{l}\text { entwickelten } \\
\text { Parabraunerde }\end{array}$ \\
\hline 6 & I Bv & verbraunter Horizont & \\
\hline 7 & $\mathrm{I} \mathrm{Cv}$ & kalkhaltiger primärer oder umgelagerter Löß & \\
\hline 8 & I fBv, $\mathrm{t}$ & intensiv verbraunter Boden des Stillfried-B-Interstadials & \\
\hline 9 & $\mathrm{II} \mathrm{Cv}$ & Gelisolifluktionsdecke aus umgelagertem Buntsandstein-M & aterial \\
\hline 10 & $\mathrm{~K} 3$ & rotbraunes, lehmiges Sediment & \\
\hline 11 & $\mathrm{~K} 2$ & spätmittelalterliche, schluffig-lehmige Kerben & \\
\hline 12 & $\mathrm{~K} 1$ & graubraunfleckiges, schluffiges, spätmittelalterliches Sedim & ent mit Holzkohle \\
\hline 13 & $\mathrm{MNZ}$ & schwach humoses, schluffig-lehmiges, homogenes neuzeitl & iches Kolluvium \\
\hline 14 & MSM Al & $\begin{array}{l}\text { spätmittelalterliches, grauhumoses, schluffig-lehmiges Koll } \\
\text { alterlichem Tonverarmungshorizont }\end{array}$ & uvium mit spätmittel- \\
\hline 15 & MSM & $\begin{array}{l}\text { spätmittelalterliches grauhumoses, schluffig-lehmiges Koll } \\
\text { (akkumuliert im frühen } 14 . \mathrm{Jh} \text {.) }\end{array}$ & ivium \\
\hline $16 \mathrm{a}$ & MHM & Grauhumoses, schluffig-lehmiges Kolluvium, akkumuliert & im 13. Jh. \\
\hline $16 \mathrm{~b}$ & MHMAh & $\begin{array}{l}\text { Zustand nach Bildung eines Humushorizontes im hochmit } \\
\text { vium } 16 \mathrm{a}\end{array}$ & telalterlichen Kollu- \\
\hline $\begin{array}{l}16 \mathrm{c} \\
\text { bzw. } 16\end{array}$ & MHMAhAl & $\begin{array}{l}\text { Zustand im Spätmittelalter: Tonverarmung im } \\
\text { hochmittelalterlichen Kolluvium 16a nach Akkumulation }\end{array}$ & von 14 und 15 \\
\hline 17 & Al in Keilen & tonverarmte Keile und Zapfen & \\
\hline 18 & g,s Tonbeläge & graue und schwarze Humus-Tonbeläge & \\
\hline 19 & $\mathrm{KH}$ & $\begin{array}{l}\text { Kulturhorizont; }-11 \text { bis }+37 \mathrm{~m} \text { Profillänge: } 9 . \text { bis } 15 . \mathrm{Jh} \\
\text { Länge: spätmittelalterlich }\end{array}$ & ; +58 bis $+65 \mathrm{~m}$ \\
\hline 20 & SG & Siedlungsgruben & \\
\hline 21 & Grab & isolierte Gräber am Ostrand des Friedhofes & \\
\hline 22 & Abbruchschicht & Abbruchschicht der Kirche (15. Jh.) & \\
\hline
\end{tabular}


neuzeitlichen Klimageschichte konnten die Aufschlüsse bei Drudevenshusen nicht geben.

\subsection{Datierung, Ausmaß, Verbreitung und Folgen der aus Bodenprofilanalysen abgeleiteten jungholozänen Extremereignisse}

Palynologische Untersuchungen der mitteleuropäischen Vegetationsgeschichte und archäologische Forschungen zeigen, „daß am Ende der römischen Kaiserzeit im freien Germanien eine Epoche riesiger Wiederbewaldungen begann, die bis ins frühe Mittelalter . . . andauerte" (ENNEN \& JANSSEN 1979: 112). Für den Zeitraum von der späten Römischen Kaiserzeit bis zum Frühmittelalter sind, da in bewaldeten Einzugsgebieten praktisch keine Bodenerosion auftritt, keine aus Bodenprofilen ableitbare Aussagen über hygrische Extremereignisse möglich.

Die frühmittelalterlichen Rodungen beendeten die geomorphodynamische Stabilitätsphase mit Bodenbildung und ermöglichten eine geomorphodynamische Teilaktivität i. S. von ROHDENBURG (1971). Exakte Vorstellungen über das Ausmaß der früh- und hochmittelalterlichen Bodenerosion am Hang bestehen nur für wenige südniedersächsische Standorte (Aufschlüsse bei Rüdershausen, Drudevenshusen, Wendeleveshusen, vgl. BORK 1983: $37 \mathrm{ff}$., Coppengrave, vgl. BORK 1985 b: $269 f$ f.). Basierend auf der oben beschriebenen Rekonstruktionsmethode wurden für diese Profile mittlere früh- und hochmittelalterliche jährliche Hangabtragsraten zwischen 8 und 20 t/ ha berechnet, Werte um $10 \mathrm{t} / \mathrm{ha}$ dominierten. Lineare Bodenerosion konnte - außer in einem Hohlweg bei Wendeleveshusen - für diesen Zeitraum nicht nachgewiesen werden. Die gefundenen früh- und hochmittelalterlichen Kolluvien waren stets ungeschichtet. Es ist daher sehr wahrscheinlich, daß die Akkumulationshöhe zwischen zwei Bodenbearbeitungen in keinem Fall höher als die Bodenbearbeitungstiefe war. Aus diesen Informationen und den vergleichsweise sehr geringen Abtragswerten ist zu folgern, daß im Früh- und Hochmittelalter nur schwach erosive Niederschläge auftraten.

Diese Phase schwach erosiver Niederschläge wurde im frühen Spätmittelalter unterbrochen. Auf zahllosen ackerbaulich genutzten Hängen Mitteleuropas rissen mehr als $10 \mathrm{~m}$ tiefe Schluchten ein. In kleineren Tälern schnitten sich mehrere Meter tiefe Kerben ein. Durch starke Lateralerosion entstanden in diesen Talauen teilweise mehrere Zehnermeter breite Talbodenpedimente. Vielerorts verursachte extrem starker Oberflächenabfluß die hangwärtige Rückverlegung der Kerbenwände und damit die Bildung von Hangpedimenten. Diese Abtragsformen wurden von den
Verfassern in verschiedenen mitteleuropäischen Landschaften beobachtet. Detailliertere Untersuchungen konnten bislang in Niedersachsen und Hessen realisiert werden. Hier wurden neben den beschriebenen Aufschlüssen in weiteren acht Profilen Sedimente datiert, die Kerben und Pedimente plombieren. Abb. 1 zeigt die Lage dieser Profile. Die Füllungen wurden ohne Ausnahme in das Spätmittelalter eingeordnet. Für die Profile bei Drudevenshusen und Coppengrave gelang eine genaue Datierung der Sedimente, die unmittelbar vor u n d unmittelbar nach dem Kerbenreißen abgelagert wurden: Die jüngsten vor den Erosionsereignissen akkumulierten Kolluvien wurden in das 13. Jh. und die Basis der Füllungen in das frühe 14. Jh. datiert. Bei den übrigen acht Profilen konnte dagegen nur die Füllung datiert werden, nicht aber der exakte Zeitpunkt der Einschneidung. Um das Alter der linearen Erosion für diese Standorte abschätzen zu können, wurden die Kerbensedimente analysiert. Die Frage nach der Geschwindigkeit der Verfüllung wurde bereits am Beispiel des Profils Rüdershausen ausführlich beantwortet. Hier und in anderen Profilen führten von den steilen Kerbenwänden abrutschende Blöcke zur raschen Plombierung der Erosionsformen. Ein weiterer Beleg dafür, daß zwischen Einschneidung und datiertem Abschnitt der Füllung nur eine extrem kurze Zeitspanne lag, ist die fehlende Bodenbildung an der Kerbenoberfläche bei sämtlichen in Abb. 1 genannten Profilen. Es liegt daher nahe, für die Bildung dieser zehn $z$. T. weit voneinander entfernten Erosionsformen einen kurzen, höchstens wenige Jahre umfassenden Zeitraum und dieselben extremen Witterungsereignisse anzunehmen.

Da vergleichbare Formen und Sedimente a u ch in anderen mit teleuropäischen Landschaften beobachtet wurden, kann von e iner Zerschluchtung aller re liefierten und ackerbaulich ge nutzten Landschaften Mitteleuropas durch we nige Katastrophenregen Anfang des 14. Jahrhunderts ausgegangen werden.

Anthropogene Einflüsse, die zur Zerschluchtung ganzer Landschaften in einem oder wenigen Jahren führten, sind nicht vorstellbar. Hingegen ist vielerorts die verheerende Wirkung der spätmittelalterlichen Zerschluchtung auf die Agrarstruktur nachweisbar. So fielen viele Kerben mit ihrer näheren Umgebung wüst und blieben bis heute bewaldet. Die starke Zerschneidung wurde von intensiver flächenhafter Bodenerosion begleitet, die hinsichtlich ihres Ausmaßes und ihrer Wirkung für die gesamte historische Zeit ihres- 
gleichen sucht. So vollzog sich ein erheblicher Teil der jungholozänen flächenhaften Bodenumlagerungen in der ersten Hälfte des 14. Jahrhunderts.

Die Folgezeit war durch schwache flächenhafte Bodenerosion geprägt, die zur vollständigen Plombierung der meisten Kerbtäler und Pedimente noch im Spätmittelalter führte: Viele schwach erosive und wenige mäßig stark erosive Niederschläge dürften diese Entwicklung verursacht haben, die sich bis in das 17. Jh. fortsetzte. Eine zweite Zerschneidungsphase führte im 18. Jh. zur Eintiefung schmaler, häufig über $4 \mathrm{~m}$ tiefer Kerben an den Tiefenlinien und zur Einschneidung bis zu $5 \mathrm{~m}$ tiefer Runsen auf beackerten Hängen. Die Ausraumvolumina erreichten allerdings nur etwa 10 bis $30 \%$ der spätmittelalterlichen Werte. Versturzmassen in den Füllungen belegen eine rasche Verfüllung und damit auch eine schnelle Eintiefung. Diese Einschneidung kann wiederum nur auf Starkniederschläge zurückgeführt werden. Agrarstrukturelle Veränderungen kommen aus den o. g. Gründen nicht als Ursache für diese zweite Phase linearer Bodenerosion in Frage (vgl. BORK 1983: 59ff.). Zur Absicherung der Datierung sind weitere Untersuchungen erforderlich. Die nach dieser zweiten Zerschneidungsphase aufgetretene Bodenerosion dürfte mit der heutigen vergleichbar sein. Schwach erosive Niederschläge lösten schwache flächenhafte Bodenumlagerungen aus. Kerben des 18. Jahrhunderts blieben häufig unter Wald erhalten. Eine Auffrischung dieser Kerben wird heute vereinzelt und auch dann nur durch Fremdwasser oder durch austretendes Grund- oder Hangwasser ausgelöst.

\section{Hygrische Extremereignisse nach Literaturangaben}

Neben den erwähnten sedimentologisch-bodenkundlich-paläoökologischen, durch archäologische Befunde datierten Zeugnissen sind in der Literatur zahlreiche andere Quellen zur jungholozänen Klimageschichte genannt und diskutiert (vgl. FLOHN 1958, 1967). Die anhand von Bodenprofilanalysen nachgewiesene spätmittelalterliche Starkregenphase kann durch instrumentelle und systematische nicht-instrumentelle Aufzeichnungen nicht überprüft werden, die Phase des 18. Jahrhunderts nur sehr eingeschränkt. Hinweise auf exzessive hyg $\mathrm{r}$ is $\mathrm{ch}$ e Witterungsereignisse vermögen Chroniken zu geben. Allerdings müssen diese chronikalischen Informationen außerordentlich kritisch analysiert werden. Eine Auswertung der schriftlichen Erwähnungen von hygrischen Extremereignissen wird durch die Zusammenstellung von Chroniken zur Witterungsgeschichte von WEIKINN (1958) sehr erleichtert.

\subsection{Mittelalter}

WEIKINN (1958) erwähnt 1391 Hinweise auf Flußüberschwemmungen, Oberflächenabfluß und Bodenerosion in Mitteleuropa während des Mittelalters. Für das frühe und hohe Mittelalter fehlen Erwähnungen ganz ungewöhnlicher Überschwemmungen - was „man nicht a priori als Beleg für ihr Nicht-Vorkommen werten" kann (FLOHN 1967: 83).

Mehr als ein Drittel der von WeIKINN (1958) zitierten 1146 spätmittelalterlichen Quellenangaben erwähnen Extremereignisse, die sich in $\mathrm{nur}$ a ch t $\mathrm{J}$ a h r e $n$ ereigneten. Mehr als 100 Schriftquellen beschreiben die Überschwemmungen des Jahres 1342. Die Jahre 1343 (34 Erwähnungen), 1374 (59), 1432 (58), 1433 (32), 1480 (52), 1491 (42) und 1496 (43) werden ebenfalls häufig genannt. Das alle anderen Hochwasser des Mittelalters und der Neuzeit an Ausmaß weit übertreffende Ereignis ist das vom 19. bis 25. Juli 1342 (vgl. z. B. FLOHN 1967: 81). WEIKINN (1958: 202-216) zitiert 64 Quellen, die die für die gesamte historische Zeit einmaligen Auswirkungen dieses Ereignisses beschreiben. Der Verlauf des Ereignisses kann, basierend auf den Angaben in WEIKINN (1958), wie folgt rekonstruiert werden: Am 19. und 20. Juli 1342 werden katastrophale Schäden in Mittelund Oberfranken erwähnt. Die Hochwasser erreichen am 21. Juli Würzburg und Frankfurt a. M., am 24./25. Juli Köln und die Niederlande. In Frankfurt dauern die Überschwemmungen bis zum 24. Juli an. Der Main erreicht hier mit $785 \mathrm{~cm}$ über dem Nullpunkt den höchsten bekannten Wasserstand (WEIKINN 1958: 204), der zweithöchste Stand wird um mehr als 2 m übertroffen (FLOHN 1967: 81). In Thüringen und Sachsen werden am 21. Juli Katastrophenregen registriert. Die resultierenden Hochwässer erreichen am gleichen Tag die Elbe bei Meissen, am darauffolgenden Tag die untere Werra sowie die Weser. Auch in anderen Gebieten wurden starke Schäden registriert. Die Hochwasser zerstörten die berühmten Brücken von Regensburg, Würzburg, Frankfurt und Dresden (FLOHN 1967: 81). Formulierungen wie z. B. ,Wasser sprudelt aus den Gipfeln der Berge', ,Wasser bedeckt Gegenden, wo es ungewöhnlich war', ,Wasser brach aus verborgenen Orten in den Bergen in Strömen hervor' und ,Gießbäche strömten aus der Erde' (WEIKINN 1958) weisen auf ganz ungewöhnlich starken Oberflächenabfluß hin. Die Bemerkung, daß alle unterirdischen Wasserquellen zerbrochen wurden (WEIKINN 1958: 210) bestätigt den auf Bodenprofilanalysen beruhenden Befund, daß Dellen, Quellbereiche und Vorfluter durch exzessive Bodenerosion zerrunst wurden.

Studien von FlOHN (1967), LAMB (1977) und anderen zeigen, daß nicht nur das Jahr 1342, sondern auch 
andere Jahre im frühen 14. Jh. durch außergewöhnliche hygrische Witterungsextrema geprägt waren. So berichtet LAMB (1977: 145), daß in den Jahren nach 1300 in den meisten Teilen Europas das bei weitem häufigste Auftreten von Hochwasserkatastrophen der letzten 2000 Jahre verzeichnet wurde. FLOHN (1967: 87) beschreibt den Zeitraum von 1310 bis 1350 als ungewöhnlich regenreich in Mittel-, West- und Osteuropa. Anormal nasse Sommer mit Überschwemmungen verursachten von 1313 bis 1317 in vielen $\mathrm{Ge}$ bieten Europas Mißernten und Hungersnöte (LAMB 1977: 451). Katastrophale Bodenerosion könnte die unmittelbare Ursache dieser Agrarkrise gewesen sein. Zusammenfassend kann festgestellt werden, daß die schriftlichen Überlieferungen die Resultate der Bodenprofilanalysen bestätigen.

\subsection{Neuzeit}

Nach spärlichen schriftlichen Überlieferungen in der frühen Neuzeit häufen sich in der zweiten Hälfte des 18. Jahrhunderts wieder Meldungen über extreme Starkregen, starke Hochwasserschäden sowie Bodenerosion. Sie bestätigen damit die Resultate der Bodenprofilanalysen, die für das 18. Jh. eine rasche Entstehung von Runsen ergaben.

Eine Zusammenstellung der Schriftquellen, die über Starkregen und ihre Folgen im Eichsfeld von 1600 bis 1930 berichten, ist in BORK (1983: 58) wiedergegeben. Danach fehlen aus den Jahren vor 1744 und von 1793 bis 1849 Hinweise auf Unwetter weitgehend, während für den Zeitraum von 1744 bis 1792 zahlreiche extreme Witterungsereignisse für den erwähnten Raum belegt sind. Vermehrte Schadensmeldungen seit der Mitte des 19. Jahrhunderts beruhen dagegen vornehmlich auf einer vollständigeren Dokumentation der schwächeren Starkregen.

Einige Beispiele mögen das Ausmaß der Zerstörungen in der zweiten Hälfte des 18. Jahrhunderts verdeutlichen. JACOBI beobachtete Mitte des 18. Jahrhunderts am Harz und in Hessen das Einreißen tiefer Gräben und daß ,in einem Menschenalter in seiner Heimat vormals gute Äcker von Erde vollkommen entblößt" wurden (HEMPEL 1957: 12). Starke Bodenerosion ist z. B. für die Gemarkung Hilkerode im Untereichsfeld belegt, in der 1776 etwa $35 \%$ der Oberfläche zerschluchtet war. PFISTER (1975: 81ff.) berichtet über Witterungskatastrophen von 1768 bis $1771 \mathrm{im}$ Schweizer Mittelland, vON RUDLOFF (1967: 134ff.) von vermehrten Hochwassermeldungen im Dezennium 1784 bis 1793 und von einem Rheinhochwasser, das alle späteren Überschwemmungen um 2 bis $3 \mathrm{~m}$ übertraf.

Archivstudien von HARD (1970) ergaben für die westliche Pfalz, das Saarland und Lothringen ein Maxi- mum der linearen Bodenerosion zwischen etwa 1760 und 1850. HARD führt die beobachteten Schäden auf agrarstrukturelle Veränderungen und nicht auf klimatische Schwankungen zurück. Die Begrenzung des Schluchtenreißens auf wenige Jahre kann nicht auf Agrarstrukturveränderungen zurückgeführt werden, da die Bodenanalysen auf besonders rasches Kerbenreißen und außergewöhnlich heftige Starkregen hinweisen (vgl. dazu BORK 1983: 59-61).

Während über den Verlauf der spätmittelalterlichen Starkregen- und Bodenerosionsphase recht genaue Vorstellungen bestehen, sind für die zweite Phase Zeitpunkt und Intensität der Starkregen noch weitgehend unbekannt. Wahrscheinlich waren die Starkregen des 18. Jahrhunderts deutlich schwächer als die spätmittelalterlichen.

\section{Schlußfolgerungen und Ausblick}

Die Detailanalyse komplexer Sediment-Boden-Aufschlüsse hat sich als einsetzbar zur Rekonstruktion des Ausmaßes und des ungefähren Zeitraumes exzessiver linearer und flächenhafter Bodenerosion erwiesen. Dieses Verfahren erlaubt darüber hinaus Rückschlüsse auf die hygrischen Witterungsereignisse, die die Zerrunsung auslösten und die zur Verfüllung der Erosionsformen führten. Schriftliche Quellen bestätigen die anhand von Aufschlußanalysen identifizierten Starkregen- und Erosionsphasen des 14. und 18. Jahrhunderts.

Es ist vorgesehen, nach den erfolgreichen Grabungen in Südniedersachsen und Hessen das Untersuchungsgebiet auszudehnen, um auch in anderen mitteleuropäischen Landschaften den Nachweis exzessiver Bodenerosion zu führen.

\section{Danksagung}

Herrn Prof. Dr. H. RoHDEnBuRG, Braunschweig, und Herrn Dr. H. -G. STEPHAN, Göttingen, danken wir für ihre Unterstützung und die hervorragende Zusammenarbeit ganz herzlich.

\section{Schriftenverzeichnis}

Bork, H.-R. (1983): Die holozäne Relief- und Bodenentwicklung in Lößgebieten. - Catena Supplement, 3: 1-93; Braunschweig.

- (1985a): Untersuchungen zur nacheiszeitlichen Relief- und Bodenentwicklung im Bereich der Wüstung Drudevenshusen bei Landolfshausen (Landkreis Göttingen). - Nachr. Nieders. Urgeschichte, 54: 59-75; Hildesheim. 
BORK, H.-R. (1985 b): Mittelalterliche und neuzeitliche lineare Bodenerosion in Südniedersachsen. - Hercynia N.F., 22/3: 259-279; Leipzig.

ENNEN, E. \& JANSSEN, W. (1979): Deutsche Agrargeschichte: vom Neolithikum bis zur Schwelle des Industriezeitalters. - Wissenschaftliche Paperbacks Sozial- und Wirtschaftsgesch. 12: IX + 273 S.; Wiesbaden (Steiner).

FLOHN, H. (1958): Klimaschwankungen der letzten 1000 Jahre und ihre geophysikalischen Ursachen. Deutscher Geographentag Würzburg, Tagungsbericht und wiss. Abh.: 201-214; Wiesbaden (Steiner).

- (1967): Klimaschwankungen in historischer Zeit. In: voN RudLoff (Hrsg.): Die Schwankungen und Pendelungen des Klimas seit Beginn der regelmäßigen Instrumenten-Beobachtung: $\quad 81-90 ; \quad$ Braunschweig (Vieweg).

HARD, G. (1970): Exzessive Bodenerosion um und nach 1800. - Erdkunde, XXIV: 291-308; Bonn.

Hempel, L. (1957): Das morphologische Landschaftsbild des Unter-Eichsfeldes unter besonderer Berücksichtigung der Bodenerosion und ihrer Kleinformen. Forsch. z. Dtsch. Landeskunde, 98: 55 S.; Remagen.

LAMB, H. H. (1977): Climate - present, past and future. 2: Climatic history and the future. $-\mathrm{XXX}+835 \mathrm{~S}$.; London (Methuen).
PFISTER, C. (1975): Agrarkonjunktur und Witterungsverlauf im westlichen Schweizer Mittelland 1755-1797. Geographica Bernensia, G2: 1-229; Bern.

ROHDENBURG, H. (1971): Einführung in die Klimagenetische Geomorphologie. - XV + 352 S.; Gießen (Lenz).

Rudloff, H. voN (1967): Die Schwankungen und Pendelungen des Klimas in Europa seit Beginn der regelmäßigen Instrumenten-Beobachtungen (1670). - $355 \mathrm{~S}$.; Braunschweig (Vieweg).

StePhan, H.-G. (1985): Ergebnisse und Perspektiven archäologischer Forschung zur mittelalterlichen Besiedlungsgeschichte des Unteren Eichsfeldes. - Nachr. Nieders. Urgeschichte, 54: 31-57; Hildesheim.

WeIKINN, C. (1958): Quellentexte zur Witterungsgeschichte Mitteleuropas von der Zeitwende bis zum Jahre 1850 , I: Hydrographie, 1: Zeitwende bis 1500. - VII + 531 S.; Berlin (Akad. Verlag).

Manuskript eingegangen am 26. 6. 1986, Nachträge August 1986. 